\title{
Would Cholera become a Neglected Tropical Disease?
}

\author{
Anil K. Bholaa , Ananda Amarasinghe ${ }^{\mathrm{b} *}$ \\ aIndependent Public Health Consultant, New Delhi, India. ${ }^{b}$ Epidemiology Unit, Ministry of Health, Colombo, Sri Lanka
}

\section{A B STRACT}

Cholera is a serious public health problem, particularly in Africa, Asia, South and Central America. Similar to the neglected tropical diseases (NTD), it is common in places with unsafe water, poor sanitation and limited access to the basic health care. In the concurrent times when the world is shrinking into a Global village, the definition of NTD itself has lost significance owing to rapid transmission of such diseases. Even, the cholera non-endemic developed countries in Europe, Russia and Oceania have become vulnerable to imported cholera. The seventh pandemic of cholera is in its $51^{\text {st }}$ year without any sign of abatement despite improvements in sanitation, safe drinking water supply, promotion of oral rehydration therapy and availability of alternative preventive interventions like oral cholera vaccines. Cholera is markedly under reported due to a myriad of reasons including economic ones. The under reporting is also resulted from weakening surveillance system across countries which in turn contributes to changing perceptions and attitudes on burden of the disease and deteriorating public health services. In addition to this, lack of evidence-based sector wide programmatic approach and inequitable resource allocation for prevention and control of cholera may also risk the disease to become a NTD in future.

Key Words: cholera, disease burden, neglected tropical disease

\section{INTRODUCTION}

More than 1 billion people are at risk of being infected by one or more neglected tropical diseases (NTDs), and approximately 534, 000 deaths occur annually as a result of NTDs. ${ }^{1}$ These are named neglected because these diseases persist exclusively in the poorest and the most marginalized communities, and have been largely eliminated elsewhere and thus are often forgotten. The affected people are less empowered and articulated to express their health needs.

In the concurrent times, the definition of neglected disease itself has certain limitation. The spread and transmission of infectious diseases across the political borders in the world can happen within hours. Whilst the definition of neglected tropical diseases says that such diseases do no travel easily, it is recognized that it is possible to travel between most places in the world in less time than the incubation period for many infectious diseases henceforth facilitating transmission of the infection through carriers. ${ }^{2}$

*Address for correspondence:

Fax: +94 112696583

E-mail: ana_amarasinghe@yahoo.co.uk

DOI: 10.5530/ijmedph.3.2011.2
The World Health Organization (WHO) does not yet categorize cholera in the list of neglected tropical diseases although it shares common traits. ${ }^{3}$ The geographic distribution of cholera is similar to neglected tropical diseases. ${ }^{4}$ Cholera disease is most commonly affecting marginalized people living in settings with limited access to safe drinking water, sanitation and basic health care. It is an intestinal disease characterized by profuse watery diarrhoea and usually associated with vomiting; leading to severe dehydration. If untreated, severe dehydration and resulting complications; such as renal failure, shock, hypokalemia, and pulmonary oedema can lead to death in $60 \%$ of cases or more, with most deaths occurring during the first day. ${ }^{5}$

In this review paper, we have explored the facts that may potentially lead cholera to be a NTD, possibly in near future. We have identified three broad reasons to justify our hypothesis; weakening disease surveillance, lack of evidence based sector wide programmatic approach in prevention and control and inequity in resource allocation.

\section{CHOLERA DISEASE BURDEN}

The cholera disease burden is characterized by both endemic disease and epidemics. Endemic cholera is defined as "occurrence of cholera outbreaks that exist predictably 
every year or every other year over a period of 5-10 years and that are recurrent in time and place." ${ }^{.6}$ Epidemics occur when the disease is introduced in an area with low or no endemicity, and thus causing unexpected and sudden surge in the number of cholera cases in a particular area in a specific time. According to the WHO, "almost every developing country reported of having either a cholera outbreak or at risk of an epidemic."

The world is currently experiencing its seventh pandemic with the first one believed to be originated in the lower Ganges delta' in 1817. 'The first six pandemics originated in Bengal, popularly known as 'home of cholera' between 1817 and 1925. While the seventh pandemic started in Indonesia in 1961 and is still continuing (Figure 1). The causative organism, $V$. cholerae $\mathrm{O} 1$ biotype El Tor, has since spread worldwide, replacing the classical strain as the leading cause of endemic cholera. This pandemic led to the re-emergence of cholera in Africa in 1970, and South and Central America in 1991, after the absence of cholera for more than a century. ${ }^{5}$ Later in 1992, a new serogroup $V$. cholerae $\mathrm{O} 139$ emerged in Bangladesh and spread to India and Pakistan. A new hybrid strain of $V$. cholerae $\mathrm{O} 1$ has replaced the original El Tor strain of the seventh pandemic in Bangladesh in 2001. ${ }^{8}$ These hybrid strains are now predominant in parts of Africa, South and Southeast Asia and are spreading globally. ${ }^{\text {The }}$ There concerns that the O139 serogroup could cause an eighth pandemic. However, the number of cases of cholera caused by this serogroup remains a small proportion of the total cases of cholera. ${ }^{5,10}$
Since 2000 , annually 95,000 to 237,000 cholera cases and 1,900 to 6,300 cholera-related deaths have reported to the WHO from around 50 countries. ${ }^{4}$ There has been a shift of reported cholera from Asia to Africa and; South and Central America in a past few decades (Figures 1 and 2). Despite low reporting, the officially reported cases from Africa has increased considerably from $20 \%$ of global reported number in the 1970 s to $94 \%$ in the period 2000-2005 whilst the Asian share has simultaneously dropped from $80 \%$ to $5.2 \%$ over the same three decades. ${ }^{11}$ Similar scenario can be noted in reported deaths. Africa share has increased from $22 \%$ to $97 \%$ of cholera deaths in global, and Asian share has showed a steep decline from $77 \%$ to $2.4 \% .^{12}$

The reported burden of Cholera may be just a tip of the iceberg and does not really reflect the true burden of cholera at population level. The WHO acknowledges that only around $1 \%$ of cholera cases are actually reported. ${ }^{11}$ Cases of cholera often remain undetected or unreported for various reasons. Health advice is commonly not sought when symptoms are mild, and stools may not be routinely cultured for $V$. cholerae. ${ }^{5,13}$ Besides, limitation in the surveillance and reporting system, fear of negative impact on tourism and trade also contribute to underestimates of the prevalence of cholera, especially in developing countries.

\section{Africa}

The most severe and deadly cholera epidemic in recent history occurred among Rwandan refugees influx into Goma,

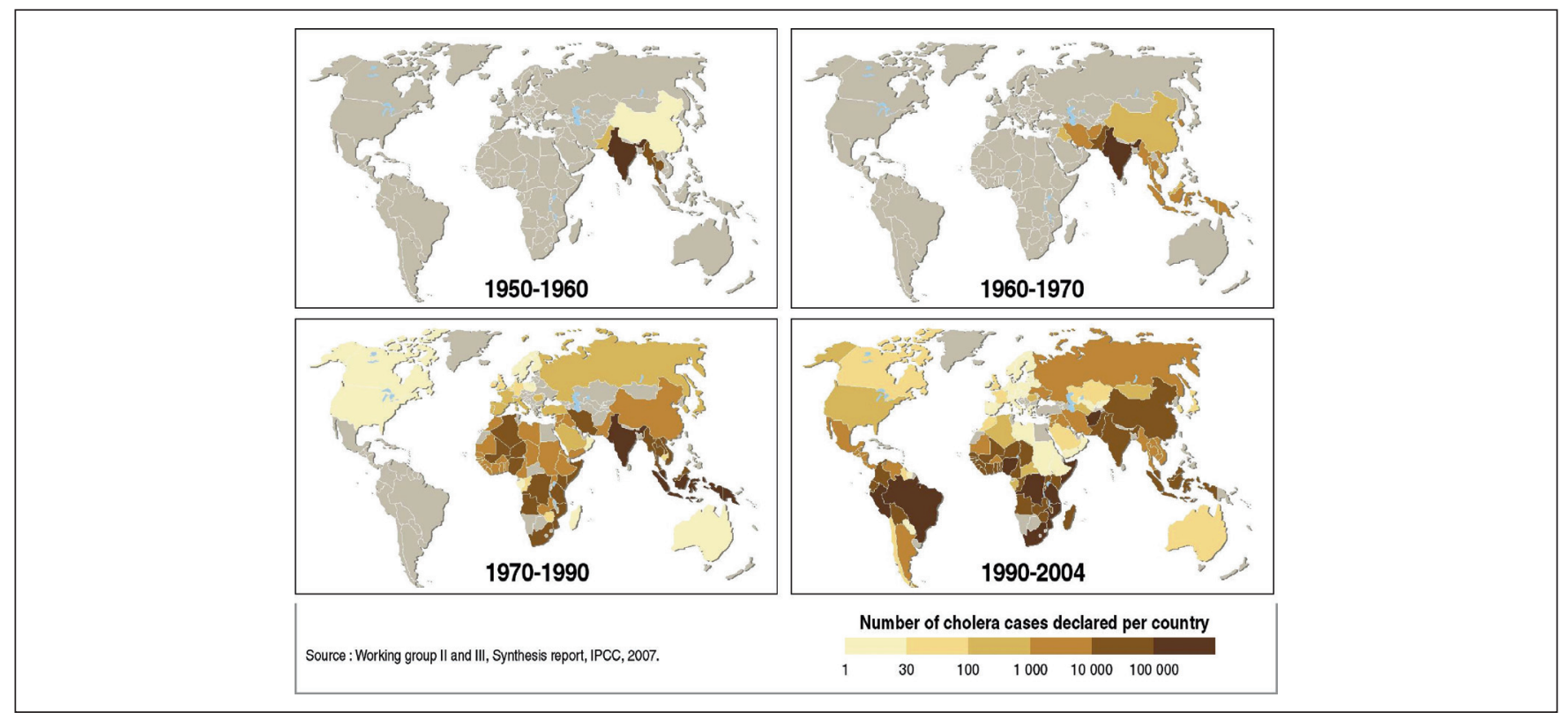

Figure 1: Global map on distribution of cholera burden across regions

Source and courtesy: UNEP/GRID-Arendal. The spread of cholera 1950-2004. UNEP/GRID-Arendal Maps and Graphics Library. 2009. Available at http://maps.grida.no/go/graphic/the-spread-of-cholera-1950-2004. Accessed January 14, 2011 


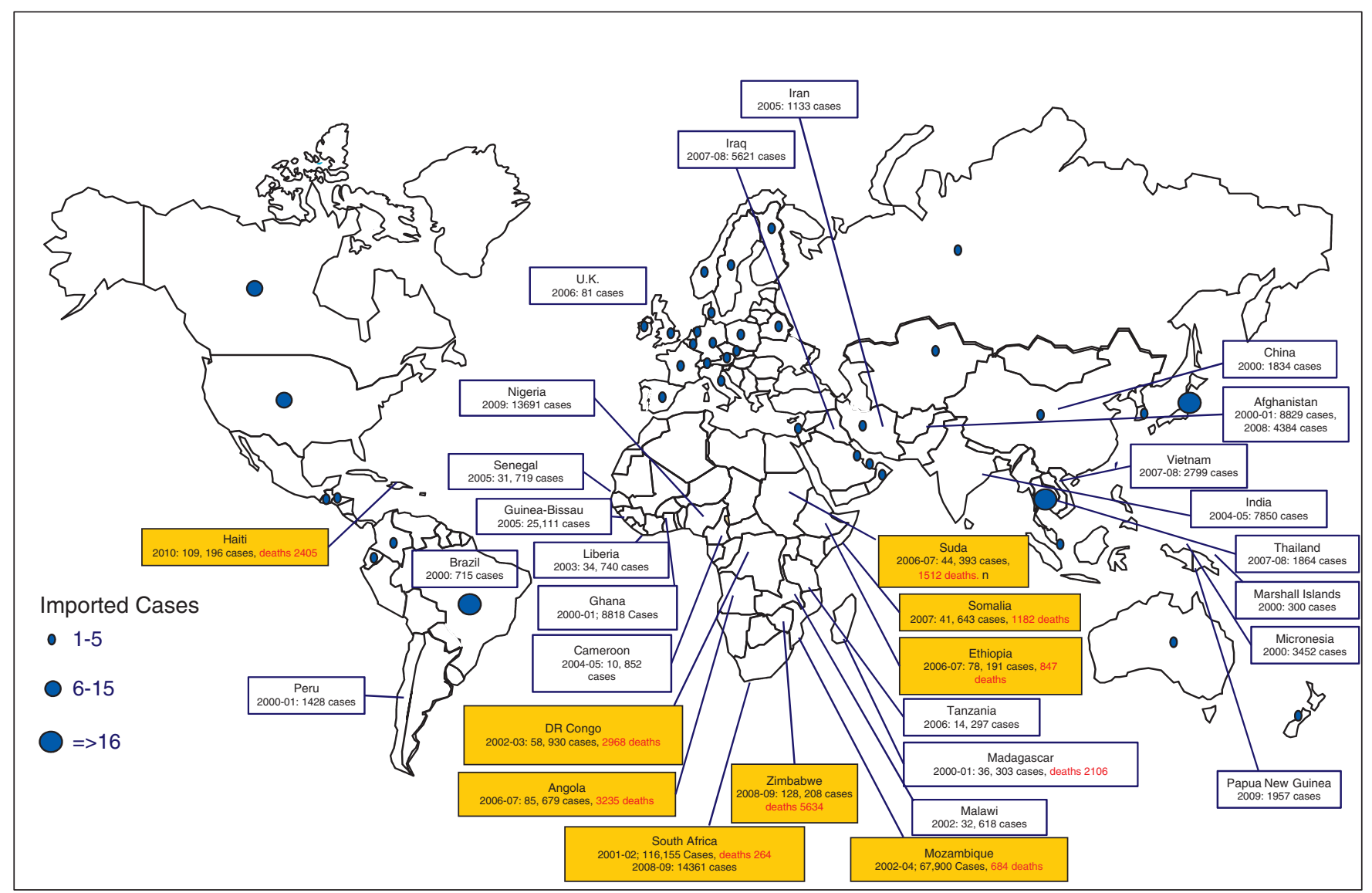

Figure 2: Cholera cases per outbreaks reported to the WHO during 2000-2010

Zaire in 1994 . Nearly $10 \%$ of refugees with an estimated 58,000-80,000 suffered from cholera. The explosive epidemic of cholera caused by $V$. cholerae $\mathrm{O} 1$ and Shigella dysentery type 1 caused death of almost 50, 0000 refugees at rate of 20-35 per 10, 000 per day. ${ }^{14}$

In 2006, Africa has reported to WHO a total of around 234, 000 cases with a Case Fatality Rate (CFR) of 2.69\%. ${ }^{4}$ The cholera epidemic in Zimbabwe in 2008-2009, over a seven months period has reported approximately 98,000 cases. The case fatality rate (CFR) was as high as $7 \%$ early epidemic period, but later declined to $4.3 \%$ due to a major international response. ${ }^{15}$ However, this cholera epidemic spread to four more neighboring countries indicating potential risk of cross boarder spread of the disease if interventions are not timely and effective.

Out of 36 sub-Saharan African countries 26 are considered as either endemic or epidemic for cholera. The total population in these 26 countries is around 980 million which is $98 \%$ of the sub-Saharan population. ${ }^{16}$ The reported incidence is ranged from 0.62 to 12.2 per 1000 persons, lowest in Democratic Republic of Congo and highest in Liberia and Zimbabwe. The actual CFR in these sub-
Saharan countries could be higher, in fact, since some people may die before reaching a health facility, especially in $2002-07 .{ }^{17}$

\section{Asia}

In Asia, 16 out of 27 countries have reported cholera cases during the period 2000-2009. In 2008, Asia reported to WHO a total of over 13000 cases though CFR remained low as of $0.64 \% .^{18}$

In Bangladesh, the seventh world highest populated country with around 160 million peoples, epidemics of diarrhea are every year phenomenon. The reported incidence of cholera is considerably varied from year to year, as $0.45 / 1000$ (in 2001) to 3.5/1000 population in 1994. Children under five years of age have by far the highest incidence rates ranging from 3 to 9 per 1000 (1994-1999). Cholera is significant burden in young children over two years of age. ${ }^{19}$ Despite cholera is not common among infants, Bangladesh has reported high rates of cholera in infants with an average of 4.6 per 1000 population over the 10-year period of 1994-2003..$^{20}$ The reported infant cases of cholera in other countries are limited and therefore true burden of cholera among infant population is unknown. 
In India, cholera is hyper-endemic in the south-eastern Indian states. Out of 35 states in India, 11 states are endemic or epidemic susceptible for cholera. ${ }^{21}$ The vulnerable population susceptible to cholera in these states is 34.5\% of total population in India, indicating the potential burden of cholera in the country. India is a fast developing country and domestic travel within states has improved remarkably. However, proportionately the other infrastructures such as sanitation and safe water supply which are closely linked with cholera have not reached to the safety levels. Increased inter states travel would have linked and caused spread of cholera outbreaks from epidemic to non epidemic states thus increasing the cholera reporting states from 11 to 21 during past 15 years period. ${ }^{21-25}$ With this Indian example, it causes concern that un-equal development within a country may also be associated with spread of the disease.

\section{Americas, Europe and Oceania}

It was the second epidemic (1826-51), 'greatest cholera pandemic of the nineteenth century' that brought cholera to Europe and America; initially reached to eastern part of Russian continent through caravan route. ${ }^{26}$ The disease reached in South America by 1991, initially in Peru and subsequently spreading to 11 countries, causing a reported 400, 000 cases and 4000 deaths within a year of its arrival on the continent. ${ }^{4}$ However, cholera was relatively quickly contained in the Americas due to concerted efforts of piped safe water supply and sanitation system, chlorination of drinking water and health education. During the period 2000-2009, almost every year there has been reported imported cases of cholera in the American region. In 2000, around 3000 imported cases were reported, but the numbers declined in subsequent years. However, in early 2010 the disease re-emerged in Haiti after almost 20 years, following the massive earthquake which completely disrupted the all basic infrastructures including drinking water supply and health care services. It was followed by flood. The poor immune status of Haiti population also became another conducive factor for the epidemic. The outbreak continued during the year and Haiti's Ministry of Health reported over 194, 000 cholera cases and nearly 4,000 deaths. Without access to treatment the mortality rate rose from $1 \%$ to as high as $25-50 \%$ for severe cases. Although, the over-all CFR was $2.0 \%{ }^{27}$

A very few imported cholera cases have been reported in the Europe (United Kingdom, Germany) ${ }^{28}$ and Oceania (Australia $^{29}$ and New Zealand) regions in last two decades. ${ }^{28,30}$ But, in Oceania, Micronesia (in 2000) and Papua New Guinea (2009) have reported large outbreaks. ${ }^{15,31}$

While comparing the inter-continental variations in the cholera burden, although it becomes obvious that only low-income countries in Africa, Asia, South and Central
America are mainly affected but developed countries in other continents are also at risk of cholera outbreaks owing to naïve immunity among present generation in those countries and to fast modern ways of disease transmission. ${ }^{2}$

The continuing high burden of cholera specifically in Asia and Africa indicate the urgent need to have a clear future vision and strong political will for concerted efforts at national and regional level for the control and prevention of cholera.

\section{CHOLERA WOULD BE A NEGLECTED DISEASE}

Like NTDs, cholera is also common in places with unsafe water, poor sanitation and limited access to the basic health care and mostly concentrated in settings of extreme poverty in remote rural areas, in urban slums or conflict zones and thrive in conditions of impoverishment. Without improving these conditions, control and prevention of cholera is unlikely. Particularly in limited resources settings, evidencebased long term interventions, such as infrastructure development relating to sanitation and safe drinking water piped supply are more appropriate, than ad hoc development activities.

The seventh cholera pandemic is now in its $51^{\text {st }}$ year and has lasted twice as long any previous epidemic. It has infected more persons than any previous pandemic and is still showing no sign of abatement. ${ }^{12}$ In mid 1970s, with the primary health care (PHC) concept, there was a significant improvement in public health services in developing countries. As a result, the disease surveillance was improved. However, subsequently PHC concept lost grip on maintaining interest and public health service including disease surveillance was deteriorated. One of contributory factors failing to control ongoing pandemic with re-emerging outbreaks is the failure in the disease surveillance. Maintaining a sustainable surveillance for any disease is always a challenge. Weakened surveillance will lead to underestimation of the disease and thereby will provide opportunity for continuation or re-emergence of the disease. As the diagnostic facilities, case management and medical care have improved, there is a reduced attention and no fearful concerns about cholera outbreaks. Thenceforth, the reporting of cholera has become relaxed and ignored. Furthermore, treatment of all diarrhoeal diseases being more or less same in the algorithms therefore this fact also dilutes the emphasis of reporting especially on cholera.

Failure in cholera disease surveillance is not only due to deteriorating public health services delivery in developing countries, but is also linked to the country economy. Existence of cholera is perceived as a negative image of any nation 
particularly in competitive global economy. To avoid such negative reflection at International level, the cholera outbreaks are often reported as 'acute watery diarrhoea' outbreaks even if these outbreaks are laboratory confirmed. Bangladesh has not reported a single case to WHO since 2000 but the International Centre for Diarrhoeal Disease Research, Bangladesh (ICDDR,B) in the capital of Dhaka estimated that it had more than 34, 000 cholera patients pass through this one institution alone in 2005 and up to 50,000 in peak years. ${ }^{17}$ It has been estimated that Bangladesh has around 300, 000 cases of cholera per annum, based on an assumed average rate of 2 per 1000 population- a number greater than all cases reported globally to the WHO in any given year. ${ }^{32}$ Similarly, many countries in the Indian sub-continent and southeast Asia do not report cholera cases to the WHO. ${ }^{33}$ Thailand has not reported any cholera case from 1994 to 2007 although a cholera epidemic occurred in southern Thailand in 1997-1998 with isolation of $V$. cholerae in five provinces. $^{34,35}$ Indonesia too has not reported any case of cholera between 1997-2004 and since 2005 though a study reported an overall incidence of $0.5 / 1000$ population in North Jakarta, Indonesia in 2001-2003. 36,37

Cholera can cause a potential economic impact to the countries. When the European Union restricted the importation of fish from four African countries (Kenya, Uganda, Tanzania and Mozambique) in 1998 in response to cholera outbreaks in 1997, the result was an estimated loss in trade of more than US $\$ 1$ billion for five-year period of 1998-2002, or US $\$ 212$ million per year for the four countries combined. ${ }^{38}$ The increasing tourism may keep on having discouragingly ignoring impact on reporting of the true burden of cholera cases under fear of losing revenues from the flourishing tourism business.

The lack of evidence-based sector wide programmatic approach in prevention and control of cholera is common practice. One way, we would describe this as a result of weakened disease surveillance system. But, in other hand, even with limited available surveillance data, little progress has shown in evidence-based decision making for designing response to prevention and control of cholera. Except a few demonstration projects, limited information is available on cost benefits analysis and impact evaluation of different interventions, particularly in cholera endemic countries in Africa. This is due to lack of sufficient evidence in decision making and resultant resource allocation.

The wide-spread misuse of antibiotics has led to the emergence of drug resistant cholera. The strains of $V$. cholerae have become resistant to several antibiotics including most commonly used ampicillin, tetracycline, furazolidone, norfloxacin and ciprofloxacin. ${ }^{39}$ Resistance to first-line antibiotics, as well as multiple-drug resistant
(MDR) $V$. cholerae, is a frequent occurrence in choleraendemic parts of the world and can greatly complicate the treatment of cholera and drive up treatment costs. Drugresistant strains cause longer-lasting, more severe illness and higher rates of secondary infection than sensitive strains. Resistance to tetracycline and doxycycline was also noted that might have contributed to the high death rates during the 1994 cholera epidemic in refugee camps in Goma, Zaire. ${ }^{39}$ In some countries where MDR strains have emerged, only fluoroquinolones (e.g., ciprofloxacin) and other more expensive antibiotics (e.g., Azithromycin) are often effective. However, yet we do not see that many cholera endemic or epidemic countries have taken these information seriously and taken appropriate actions to address the issue of misuse of antibiotics in disease treatment.

Cholera needs a both short- and long-term evidence based interventions. There is no proper evaluation of systematic use or incorporation of cholera vaccination in ongoing immunization programmes in high-risk areas. We do not believe a need for introduction of cholera vaccine into routine immunization. Our concern is that it has been used in ad-hoc manner, mostly to control impending outbreaks. It is a timely need to evaluate and conduct a need assessment for introduction of high-risk group vaccination programmes, where it is necessary. There is a need to have identified strategies in using cholera vaccine in both endemic and non endemic countries, as similar recommendations are available for many other vaccines. Despite; many new vaccine candidates with similar disease burden have been introduced , no country has yet introduced oral cholera vaccines in public health program to control endemic or epidemic cholera, with the exception of Vietnam, which has provided a low-cost, locally-produced killed whole-cell only vaccine (without the cholera toxin B subunit; named as mORC-Vax) to high-risk populations through a yearly national immunization program since 1997.

The governments of cholera endemic countries need evidence-based strategic vision for developing a multisectoral long term strategy to address the public health problem of cholera. Although, the WHO has recently started to consider the use of oral cholera vaccines as an adjunctive public health intervention in addition to improvement in water and sanitation sectors ${ }^{40}$ still there are not substantial evidences available for the developing countries' policy formulators to make a decision on either pre-emptive or reactive use of oral cholera vaccine in a real-time public health immunization programme that are already stretched with other vaccines. Immunization of adult women with this vaccine has been shown to confer herd immunity against cholera to children too young to be vaccinated. ${ }^{41}$ The only vaccine that is pre-qualified by WHO for international use is killed monovalent (O1) whole-cell rBS vaccine (Dukoral) 
which is licensed in more than 60 countries but is still mainly used as a traveler's vaccine for its cost is beyond reach to use it as a public health intervention and this vaccine also does not provide protection against serotype O139.

Finally, inequity in resource allocation is another challenge that could lead to cholera as a NTD. A policy makers survey in seven large Asian countries (Bangladesh, China, India, Indonesia, Pakistan, Thailand and Vietnam) conducted in 2005 explored opinions regarding the need for, and potential uses of new-generation vaccines-against cholera, typhoid and shigellosis. ${ }^{42}$ Majority of governmental officials viewed cholera as less of a concern because of the decrease in mortality afforded by improved case management and availability of oral rehydration solutions and have cited water and sanitation improvement as their preferred methods for sustained cholera control. ${ }^{42}$ Despite much improvement in sanitation in both Asia and Africa, yet both regions facing greatest challenge of open defecation which is $69 \%$ for sub-Saharan Africa and 64\% Southern Asia (64\%). ${ }^{43}$ Under these conditions, it is unrealistic to depend on prevention and control of cholera only thorough infrastructure development relating to water and sanitation, although it is essential and helpful. The use of oral cholera vaccine in Africa and Asia to control disease in non epidemic situation (i.e., a non-refugee or disaster situations) through mass vaccination campaigns have demonstrated wide acceptance of the vaccine with overall over $70 \%$ coverage. ${ }^{44}$ However, cost and other related logistics challenges lead the governments not to consider the use of oral cholera vaccine as a public health intervention in its multi-sectoral strategy for control of endemic cholera. ${ }^{45,46}$ The costs of cholera vaccination have varied considerably from US\$ 0.89 to 17.55 per fully immunized person. ${ }^{45,46}$

Operational research for identifying effective strategic interventions for averting and reversing the neglected cholera pandemic should be a priority. There is need to develop and implement multi-sectoral strategy with short and long term evidence-based interventions that guide the equitable allocations of resources at country level for halting the seventh epidemic of cholera so that achievement of the Millennium Development Goals can also be accelerated.

\section{CONCLUSION}

Cholera remains a serious public health problem in developing countries. A significant improvement in case management and reducing mortality through world wide use of oral rehydration therapy is the success story in cholera. However, other interventions show a little impact on the disease control and prevention and, yet no sign of abating of the ongoing seventh pandemic. Regions or populations where surveillance is weak and evidence based intervention and equitable resource allocation are not adequate, the risk of epidemics and consequent endemics remains. The disease has already become endemic in most parts of Africa, South and South-east Asia. Endemic situations and outbreaks of cholera can be prevented by providing sanitation and safe drinking water at point-of use, promoting good personal hygiene behavior, safe food handling and prudent use of available and affordable oral cholera vaccines in highlyvulnerable populations.

If the disease burden continues to be underestimated and; frequent outbreaks and rapid transmission among impoverished and vulnerable populations remain ignored and not attended appropriately then cholera would become a neglected tropical disease in near future. However, as the world is becoming a Global village, those in developed country are also at risk as their immunity is waning as the days pass by and would face the real threat if confronted with the comma shaped bacilli vibrio cholerae.

\section{FUNDING}

No funding or support to report.

\section{COMPETING INTERESTS}

No competing interest to declare

\section{ACKNOWLEDGEMENT}

We would like to thank Dr Md. Manirul Islam, Training Coordinator, Technical Training Unit at the International Centre for Diarrhoeal Disease Research, Bangladesh for reviewing the manuscript and providing invaluable advice on this paper.

\section{REFERENCES}

1. World Health Organization: Available at http://www.who.int/features/ factfiles/neglected_tropical_diseases/ntd_facts/en/index.html (accessed on January 05,2011 ).

2. Carlos Castillo-Salgado. 'Trends and directions of global public health surveillance'. Epidemiological Rev, 2010, 32:93-109.

3. World Health Organization. Available at http://www.who.int/neglected diseases/diseases/en (accessed on January 05, 2011).

4. World Health Organization. Cholera, 2006. Wkly Epidemiol. Rec, 2007, 83:269-284.

5. Sack D.A., Sack R. B., Nair G. B., Siddique A.K. Cholera. Lancet, 2004, 363:223-233.

6. Meeting on Focus on neglected tropical infectious diseases: integrating vaccines into Global Cholera Control Efforts. Annecy, France, April 14-17, 2009, organized by the Merieux Foundation.

7. WHO. Cholera, 2004. Wkly Epidemiol Rec. 2005, 80, 261-268. 
8. Nair G.B., Qadri F., Holmgren J., Svennerholm A.M., Safa A., Bhuiyan N.A. et al. Cholera due to altered El Tor strains of Vibrio cholerae 01 in Bangladesh. J. Clin Microbiol 2006; 44:4211-13.

9. Nguyen B.M., Lee J.H., Cuong N.T., Choi S.Y., Hien N.T., Anh D.D. et al. Cholera outbreaks caused by an altered Vibrio cholerae O1 EI Tor biotype strain producing classical cholera toxin B in Vietnam in 2007 to 208. J. Clin Microbiol 2009; 47 (5):1568-71.

10. WHO. Global defense against the infectious disease threat. In:Kindhauser M.K., ed. Communicable diseases 2002. Geneva: WHO, 74-79.

11. WHO. Cholera unveiled. Global Task Force on Cholera Control. http:// whqlibdoc.who.int/hq/2003/WHO_CDS_CPE_ZFK_2003.3.pdf (accessed on January 14, 2011).

12. Gaffga N.H., Tauxe R.V., Mintz E.D. Cholera: a new homeland in Africa? Am J.Trop Med Hyg, 2007, 77:705-713.

13. Steffen R., Acar J., Walker E., Zuckerman J. 'Cholera: assessing the risk of travelers and identifying methods of protection. Travel Med Infect Dis. 2003, 1:80-88.

14. Goma Epidemiology Group. Public health impact of Rwandan refugee crisis: what happened in Goma, Zaire in July 1994? Lancet 1995/Feb 11; 345:339-44.

15. WHO. Cholera, 2009. Wkly Epidemiol. Rec., 2010, 85:293-308.

16. World Population Prospects:The 2006 Revision Population Database; subSaharan Africa, http://esa.un.org/unpp/ (accessed on January 24, 2011).

17. Sack D.A., Sack R.B., Chaignat C.I. Getting serious about cholera. N. Engl J. Med 2006; 355 (7):649-51.

18. WHO. Cholera: global surveillance summary, 2008. Wkly Epidemiol. Rec., 2009, 84:309-324.

19. Bhattacharya S.K., Datta D., Bhattacharya M.K., Garg S, Ramamurthy T., Manna B, Nair G.B., Moitra A. Cholera in young children in an endemic area [letter]. Lancet; 1992, 340:1549.

20. Sack D.A. When should cholera vaccine be used in cholera-endemic areas? J Health Popul Nutr, 2003, Dec; 21(43):299-303.

21. Kanungo S., Sah B.K., Lopez A.L., Paisley A.M., Sur D. et al. Cholera in India: an analysis of reports, 1997-2006. Bull. World Health Organ; 2010, 88:185-191.

22. Gupta N., Dewan S., Saini S. Resurgence of Vibrio cholera 0139 in Rohtak. Indian J. Med Res. 2005, 121 (2):128-30.

23. Gupta D.N., Mondal S.K., Sarkar B.L., Mukherjee S., Bhattacharya S.K. An El tor cholera outbreak amongst tribal population in Tripura. J Commun. Dis. 2004, 36 (4):271-6.

24. Sengupta P.G., Niyogi S.K., Bhattacharya S.K. An outbreak of El tor cholera in Aizwal town of Mizoram, India. J Commun Dis. 2000, 32 (3):207-11.

25. Thakur J.S., Swami H.M., Dutt R., Mehta M., Gupta V. Epidemiological investigation of cholera outbreak in a periurban slum colony in Chandigarh. Indian J. Med. Sci. 2001, 55 (8):429-33.

26. Snow J. On the mode of communication of cholera, $1858 ; 2^{\text {nd }}$ ed. J. Churchil, London, England.

27. Pan American Health Organization. Cholera outbreak in Haiti, Health Cluster Bulletin-\#15, January 21, 2011 available at http://new.paho.org/ hai/index.php?lang=en (accessed on January 24, 2011).

28. UK Health Protection Agency. Statutory notifications of infectious diseases (NOIDs)-annual totals 1994 to 2005- England and Wales. http:// www.hpa.org.uk/infections/topics_az/noids/annualtab.htm (accessed on February 4, 2011)

29. Dowse G. Cholera, imported-Australia (02). ProMed-mail. Archive number 20040319.0769. March 19, 2004. http://www.promedmail.org/pls/promed/ $f ? p=2400.1202: 10232375585835111995:: N O:: F 2400 \_P 1202 \_C H E C K$ DISPLAY,F2400_P1202_PUB_MAIL_ID:X,24788 (accessed on February 8, 2011).

30. Anon. Communicable disease surveillance: highlights for $1^{\text {st }}$ to $3^{\text {rd }}$ quarter, 2004. Commun Dis Intell 2004; 28:281-534.

31. WHO. Cholera, 2000. Wkly Epidemiol. Rec. 2001, 76, 233-240.

32. Tacket C.O., Sack D.A. Cholera Vaccines. Chapter 9, In: Vaccines, $7^{\text {th }}$ edition. Edited by Plotkin S.A., Orenstein W.A., Offit P.A., Philadelphia, P.A.: WB Saunders Company, 2008.

33. Zuckerman J.N., Rombo L. Fisch A. The true burden and risk of cholera: implications for prevention and control. Lancet Infect Dis. 2007; 7:521-30.

34. WHO. Cholera in 1994. Part I. Wkly Epidemiol Rec 1995; 70:201-08.

35. Kondo S., Kongmuang U., Kalnauwakul S., Matsumoto C., Chen C.H., Nishibuchi M., Molecular epidemiological analysis of Vibrio cholerae 01 isolated during the 1997-8 cholera epidemic in southern Thailand Epidemiol Infect 2001; 127:7-16.

36. WHO. Cholera in 1997. Wkly Epidemiol Rec 1998; 73:201-08.

37. Agtini M.D., Soeharno R. Lesmana M, et al. The burden of diarrhea, shigellosis, and cholera in North Jakarta, Indonesia: findings from 24 months surveillance. BMC Infect Dis 2005; 5:89.

38. Kimball A.M., Wong K.Y., Taneda K. An evidence base for international health regulations: quantitative measurement of the impacts of epidemic disease on international trade. Rev Sci Tech 2005/Dec; 24(3):825-32.

39. Sack D.A., Lyke C., McLaughlin C., Suwanichkij V. Antimicrobial resistance in shigellosis, cholera and campylobacteriosis. WHO Report, 2001 (WHO/ CDS/SCR/DRS/2001.8).

40. WHO. Cholera vaccines: WHO position paper. Wkly Epidemiol Rec. No. 13, 2010, 85, 117-128.

41. Ali M., Emch M., Yunus M., Sack D., Lopez A.L., Holmgren J., Clemens J. Vaccine protection of Bangladesh Infants and young children against cholera: Implications for vaccine deployment and person-to-person transmission. Pediatric Infectious Disease, Jan 2008 (27)-33-37.

42. DeRoeck D., Clemens J.D., Nyamete A., Mahoney R.T. Policymakers' views regarding the introduction of new-generation vaccines against typhoid fever, shigellosis and cholera in Asia. Vaccine; 2005, 23:2762-74.

43. United Nations. The Millennium Development Goals Report 2010. Available at: http://www.un.org/millenniumgoals/pdf/MDG\%20Report\%20 2010\%20En\%20r15\%20-low\%20res\%2020100615\%20-.pdf (accessed on February 18, 2011).

44. Chaignat C.L., Monti V., Soepardi J., Peterson G., Sorensen E., Narain J. Cholera in disasters: do vaccines prompt new hopes? Expert Rev Vaccine 2008; 7(4):431-35.

45. Ministry of Health, Mozambique. http://www.misau.gov.mz/pt/epidemias endemias/colera/plano_estrategico_multisectorial_de_combate_a_ colera (accessed on March10, 2011).

46. WHO. Use of the two-dose oral cholera vaccine in the context of a major natural disaster: Report of a mass vaccination campaign in Ache province, Indonesia, 2005. http://www.who.int/topics/cholera/publications/final tsunami.pdf (accessed on March 4, 2011). 